\title{
Tangence
}

\section{Au delà du dialogisme}

\section{Wladimir Krysinski}

Numéro 51, mai 1996

Paradigmes critiques

URI : https://id.erudit.org/iderudit/025907ar

DOI : https://doi.org/10.7202/025907ar

Aller au sommaire du numéro

Éditeur(s)

Tangence

ISSN

0226-9554 (imprimé)

1710-0305 (numérique)

Découvrir la revue

Citer cet article

Krysinski, W. (1996). Au delà du dialogisme. Tangence, (51), 98-122.

https://doi.org/10.7202/025907ar d'utilisation que vous pouvez consulter en ligne.

https://apropos.erudit.org/fr/usagers/politique-dutilisation/ 


\section{Au delà du dialogisme* \\ Wladimir Krysinski}

Je raconte. Ce je n'est pas une personne imaginaire, mais le romancier. Un homme qui est instruit, amer, désillusionné. Je. Je raconte l'histoire de mon ami Achille. Mais aussi ce qui m'arrive avec d'autres personnages du roman. (Robert Musil)

En réalité, il faudrait inventer un art qui mêlerait les idées pures à la danse, les hurlements à la géométrie. Quelque chose que l'on réaliserait dans une enceinte herméneutique et sacrée, un rituel par lequel les gestes seraient unis à la pensée la plus pure, le discours philosophique à des danses de guerriers zoulous. Une combinaison de Kant et de Jérôme Bosch, de Picasso et d'Einstein, de Rilke et de Gengis Khan. Tant que nous ne sommes pas capables d'un mode d'expression aussi intégral, défendons au moins le droit de faire des romans monstrueux. (Ernesto Sabato)

La littérature ne consiste pas à accorder des droits d'auteur à certains thèmes pour certains groupes. Et pour ce qui est du risque : les vrais risques de n'importe quel artiste sont pris dans l'œuvre, dans l'acte de pousser l'œuvre jusqu'aux limites du possible, en essayant d'augmenter la somme de ce qu'il est possible de penser. Les livres deviennent bons lorsqu'ils vont jusqu'à cette limite et prennent le ris-

- Cette étude a été réalisée dans le cadre d'un projet de recherche sur La phénoménologie de la modernité (1987-1990) subventionnée par le Conseil de Recherches en Sciences Humaines du Canada ( ${ }^{\circ}$ 410-87-0631). 
que de la franchir, lorsqu'ils mettent en péril l'artiste à cause de ce qu'il a osé ou n'a pas osé artistiquement. (Salman Rushdie)

\section{Les destins du roman : au delà du dialogisme?}

Mesurés à l'aune des catégories bakhtiniennes, les changements qui se produisent à la fin du $\mathrm{xx}^{\mathrm{e}}$ siècle dans le corps discursif du roman pointent vers un dépassement ou du moins vers une relativisation du dialogisme. Ces changements révèlent par ailleurs une réelle fonctionnalité si ce n'est une prédominance du polylinguisme et de l'hybridation comme modalités discursives. Ainsi risquera-t-on la proposition suivante: les positions théoriques de Bakbtine ne permettent de saisir que partiellement la dynamique discursive des cuvres romanesques qui ont marqué ces quinze dernièrs années.

Si l'on définit le dialogisme comme le principe axiologique qui régit la construction et la fonction du roman, il faut se rendre à l'évidence: le roman abandonne la figure de l'auteur qui, tel Dostoïevski, se retranche derrière les voix, les subjectivités et les idées de ses personnages. Le geste dialogique de l'auteur s'efface derrière la position dominante de l'auteur-constructeur. Il se relativise aussi par la mise en discours du narrateur: protagoniste ou personnage, celui-ci se manifeste en outre comme instance énonciative dotée d'une forte identité textuelle. À ce propos, les observations de Guy Scarpetta sur les modalités narratives du roman contemporain et plus particulièrement sur l'art romanesque de Milan Kundera (L'immortalité, La lenteur) et de Carlos Fuentes (Christopbe et son oeuf) paraissent tout à fait pertinentes. Scarpetta souligne l'importance dans le roman contemporain d'une modalité narrative qui unit dans la même voix, et donc dans la même expression artistique, le "narrateur omniscient" et le "narrateurpersonnage ". Voici comment Scarpetta caractérise la fonction de ce discours dans La lenteur de Kundera:

Le narrateur, par toutes sortes d'indices [...] laisse entendre qu'il est assimilable à l'auteur, à Kundera, - ce qui désoriente le système: si le point de vue "omniscient" est incompatible avec l'adoption d'un narrateur-personnage, forcément limité par sa vision "humaine", il est en revanche tout à fait possible de le référer à l'auteur, s'il s'avoue comme tel. Ensuite, parce que ce 
100

narrateur-auteur ne cesse de nous rappeler (autre forme de mise en abyme) qu'il imagine ses personnages, qu'il les invente, ou qu'il les rêve (il va même parfois, comme chez Diderot encore, jusqu'à les apostropher): l'écriture ne se laisse pas oublier derrière ce qu'elle écrit, - ce qui est, pour le coup, un indice flagrant de modernité. ${ }^{1}$

Cette mixité narrative du roman est relativement fréquente depuis les années quatre-vingt. Elle infirme la dynamique dialogique identifiée et théorisée par Bakhtine autour de Dostoïevski. En fait, le narrateur-auteur-personnage omniscient, si actif dans la prose moderne, démontre que la construction du roman ne saurait faire abstraction de son constructeur.

L'analyse bakhtinienne de l'œuvre de Dostoïevski en occulte certaines caractéristiques, notamment l'importance du monologisme comme modalité narrative. Dostoïevski, metteur en scène ou plutôt constructeur du dialogisme, accomplit sans aucun doute une révolution romanesque. Mais dans la mouvance de l'hybridité discursive et structurale, sa postérité affaiblit l'impact de cette révolution. Aussi devons-nous proposer une image du roman plus riche, plus complexe et surtout plus conforme aux pratiques actuelles.

\section{La dynamique romanesque : hypothèse d'une nouvelle série-scène}

Pour saisir le roman dans ses modernités, il faut concevoir ne serait-ce qu'hypothétiquement une série ou une scène discursive suffisamment vaste pour englober des œuvres qui se différencient des modèles élaborés depuis Cervantes jusqu'à Joyce, Musil ou Broch, et qui les ont transformés. Cette série inclurait des romans publiés depuis les années 1970: Harrouda de Tahar Ben Jelloun (1973), Topographie idéale pour une agression caractérisée de Rachid Boudjedra (1975), Talismano de Abdelwahab Meddeb (1979), La pulpe de J. Andrzejewski (1980), Makbara de Juan Goytisolo (1980), Midnight's Children de Salman Rushdie (1980), Les Géorgiques de Claude Simon (1981), Paradis de Philippe Sollers (1981), L'Algarabie de Jorge Semprun (1981), Cassandre. Le récit et les prêmisses de Christa Wolf (1983), L'année de la mort

1 Guy Scarpetta, L'âge d'or du roman, Paris, Grasset, coll. "Figures", 1996, p. 267. 
de Ricardo Reis de Jose Saramago (1984), Mâttres anciens de Thomas Bernhard (1985), Les invisibles de Nanni Balestrini (1987), Christophe et son ceuf de Carlos Fuentes (1987), L'immortalité de Milan Kundera (1990), American Psycho de Bret Easton Ellis (1991), The Runaway Soul de Harold Brodkey (1991), Texaco de Patrick Chamoiseau (1992), Tout-monde d'Edouard Glissant (1993), Sostiene Pereira d'Antonio Tabucchi (1994).

Ces ouvres confirment la prédominance de l'auteur-constructeur et du narrateur subjectif aux dépens de la position dialogique de l'auteur à la Dostoïevski. C'est pourquoi le bilan du "postbakhtinisme" de David Lodge nous paraît juste:

En fin de compte, la plus grande contribution de Bakhtine à la critique contemporaine, par-delà le paradoxe historique de sa longue obscurité et de sa célébrité, est la réaffirmation actuelle de la puissance créatrice et communicative de l'écrivain. ${ }^{2}$

Ainsi reconnu, l'apport de Bakhtine permet de comprendre le rôle de l'"Intelligence Centrale" de l'auteur comme condition sine qua non de l'intelligibilité du roman, des signes qui le fondent, de ses structures et de ses fonctions. La formule de Henry James nous rappelle que la poétique du roman repose sur l'acte créateur d'un individu, concepteur de structures fonctionnelles. Ce rappel nuance la conception dialogique de l'auteur. En attribuant à Dostoïevski cette nouveauté absolue, Bakhtine semblait présupposer que le romancier se bornait à reproduire les idées en circulation et que l'Intelligence Centrale renonçait au privilège de se faire entendre dans le texte. Dans les romans cités plus haut, l'Intelligence Centrale fonctionne souvent comme une modalité spécifique de la narration (narrateur omniscient et narrateur-personnage), modalité que Guy Scarpetta identifie et rattache à la modernité. Ces romans confirment d'autres traits spécifiques de l'écriture moderne, à savoir la subjectivité, la fragmentation, l'ironie et l'autoréflexivité, que nous avons identifiés pour notre part comme les invariants discursifs et cognitifs du texte "moderne". C'est donc sur le fond de ce dispositif qu'il faudrait mesurer l'intensité et la pertinence des modernités du roman.

2 David Lodge, After Bakbtin. Essays on Fiction and Criticism, Londres et New York, Routledge, 1990, p. 7. Nous traduisons. 
102

\section{Rushdie, Fuentes, Glissant : la quête de la totalité et le jeu de l'hybride}

L'œuvre de ces trois romanciers, notamment Midnigbt's Children, Cbristophe et son cuf et Tout-monde, exprime la quête d'une totalité qui se fonde sur l'inclusion à l'infini d'univers divergents : narratifs, thématiques, langagiers, culturels. C'est le triomphe de l'hybride comme vision et composition, ensemble flou mais sciemment composite. La totalité se constitue en un paysage humain d'une grande richesse topographique. C'est un espace diversifié de récits et de discours hétérogènes déployant des univers complexes, foisonnants, d'une pertinence immédiate et universelle. Ces formes et ces messages se constituent en opérateurs de la modernité en tant qu'ils mettent en œuvre une réécriture des matériaux romanesques culturels, historiques, politiques, formels et thématiques. C'est avec ces matériaux que sont recomposés les modèles matriciels du roman, à savoir les dispositifs perspectivistes à la Cervantes et les digressions à la Sterne.

\section{Salman Rushdie: Midnight's ChILdRen}

En affirmant son identité, Salman Rushdie observe: "Tout comme des millions de gens, je suis un enfant bâtard de l'histoire. Peut-être nous tous, noirs, bruns et blancs, nous coulons les uns dans les autres, comme le remarque un de mes personnages, tels des parfums au moment de cuisiner." ${ }^{3}$ Paradoxalement, mais tout à fait dans l'esprit des avatars, c'est-à-dire du jeu des réincarnations si important dans la mythologie indienne, on peut admettre que Saleem Sinai, le narrateur de Midnight's Children, est une des réincarnations de l'auteur et, inversement, l'auteur lui-même est une réincarnation de Saleem Sinai. Ce paradoxe jette une lumière sur l'identité de la voix narrative. Indéniablement le personnage du narrateur a partie liée avec l'Intelligence Centrale du roman. Même si l'auteur n'est pas "implicité " par des renvois qui permettraient de l'identifier, il n'en reste pas moins que la vision de l'Inde pointe sans ambiguilté du côté de Salman Rushdie, commentateur de sa propre ouvre. Dans ses écrits critiques apparaissent systématiquement les notions de multitude, de mélange, de

3 Salman Rushdie, "In good faith ", Imaginary Homelands. Essays and Criticism 1981-1991, New York, Penguin Books, 1991, p. 394. À moins d'indication contraire, toutes les citations de Rushdie sont traduites par nous. 
diversité, d'hybride. En outre, Rushdie accentue l'importance des conflits inter-ethniques et inter-religieux. L'Inde, en somme, est un pays où l'exubérance du multiple ne s'accompagne pas du respect mutuel des races et des religions différentes. Telle est aussi la leçon du roman qui donne à voir un univers voué à la dégradation et à l'intensification des conflits. La narration progresse vers une négativité incontournable, comme le montre la formule "Midnight's Children" transformée en "Midnite-Confidential Club", raison sociale d'un cabaret. L'univers merveilleux de l'espoir se transforme en un lieu où se jouent les rites de l'amour commercial. Par ailleurs, ce qui semble peser sur l'Inde, c'est l'arrivée de l'Âge de l'Obscurité, "Kali Yuga". Le fatalisme de la destruction atteint même le corps de Saleem qui se dessèche et se décompose. Le roman de Rushdie est une circulation de parfums et de formes, de mythes et de croyances qui connotent la particularité et la totalité de l'univers humain de l'Inde. Le travail narratif de Saleem distribue le positif et le négatif, l'optimisme et le pessimisme. La vision semble tourner au maléfique et à l'irréversibilité du conflictuel et du mal. Pourtant, le narrateur investit son message d'espoir. C'est un espoir fragile, mais il se dessine lorsque dans son apprentissage du réel, Saleem apprend aussi à reconnaître la multitude.

Midnight's Cbildren présente une démarche narrative multiple qui ne recule devant aucun procédé, aussi invraisemblable soit-il que l'omniscience ou l'ubiquité du narrateur. Observateur et participant idiosyncratique d'un monde inépuisable de signes, celui-ci symbolise tous les ressorts culturels de la mentalité indienne où l'omniprésence des divinités et de leurs avatars, du mythe et du surnaturel, couplée avec la multitude grouillante de l'humain est vécue au quotidien. Par une série de signes et d'informations, la narration elle-même est un geste performatif qui rivalise avec celui du narrateur suprême, Schéhérazade: "Je dois travailler vite plus vite que Schéhérazade, si je dois aboutir à un sens - oui, le sens - à quelque chose ". Le narrateur se présente comme un "avaleur de vies" (a swallower of lives"), comme celui en qui "se bousculent des multitudes" (Consumed multitudes are jostling and shoving inside me "). Il cautionne une vision du monde tributaire de sa participation active à la société indienne en tant qu'un des mille et un enfants nés à minuit le 15 août 1947. Tous ces enfants sont des citoyens exceptionnels; pourvus de dons rares, ils semblent destinés à devenir maîtres du 
104

monde. Ce moment historique marque aussi l'avènement de l'espoir, car le nouveau pays s'engage dans un avenir où devraient triompher la démocratie, la justice et la liberté. Mais les choses se compliquent. Des guerres éclatent. L'Inde est affligée de conflits religieux et inter-ethniques. En somme, le roman de Rushdie montre la dégradation systématique de l'espoir. La narration, d'une rare intensité, est conduite au nom de la désillusion.

Dans ce roman, Rushdie donne la parole à un personnage qui ne cache rien de son moi, de sa psychologie ou de son histoire. Ayant souvent recours à l'auto-identification démonstrative, le narrateur brosse un portrait riche et contradictoire de luimême. Son histoire suit l'histoire moderne de l'Inde, renvoyant à des faits historiques et politiques concrets. En même temps, l'histoire de Saleem est imaginaire et fantastique, ses "actions verbales" traduisent son appartenance au monde surnaturel, divin, mythologique. Privilège de la fiction, la construction du narrateur ne s'embarrasse pas de la véridicité de ce que Saleem vit et raconte. Construction purement fictive, c'est pourtant aussi une construction cognitive en ce sens que le foisonnement de cet univers narratif est à la mesure des complexités qui sont à l'origine même de la civilisation et de la société indiennes. Il existe donc une affinité entre la construction narrative de Midnight's Children et le système de valeurs de l'auteur. Cette affinité présuppose une compréhension de l'Inde qui détermine une certaine construction du sens. Rushdie dit clairement ce qu'il voulait exprimer dans ce roman :

Je viens de Bombay, et, aussi, d'une famille musulmane. "Mon" Inde a toujours été basée sur les idées de multiplicité, de pluralisme, d'hybridité: des idées auxquelles les idéologies des communalistes ("communalists") sont diamétralement opposées. Pour moi, l'image qui définit l'Inde est la foule, et la foule par sa nature même est surabondante, hétérogène, beaucoup de choses en même temps. Mais l'Inde des communalistes n'est aucune de ces choses. ${ }^{4}$

La dernière scène du roman illustre bien ce propos. C'est le jour de l'Indépendance, le 15 août 1978. Saleem célèbre son trente et unième anniversaire. Il veut se rendre au Cachemire, mais il ne pourra pas l'atteindre. Il va devoir affronter les "foules

4 Salman Rushdie, "The Riddle of Midnight: India, August 1987", Imaginairy Homelands, op. cit., p. 32. 
aux nombreuses têtes" ("manyheaded multitudes" 5 ). La foule est décrite comme "dense", "sans frontières", "grandissante jusqu'à ce qu'elle remplisse le monde", et qui "empêche toute avancée". Le narrateur craint d'être piétiné par la foule, tout comme son fils et les mille et un "enfants de minuit". Le roman se termine sur les propos suivants :

le privilège et la malédiction des enfants de minuit, c'est d'être à la fois maîtres et victimes, de renoncer à toute vie privée, d'être aspirés par le tourbillon annihilant et de ne pas pouvoir vivre et mourir en paix. ${ }^{6}$

Le sens du roman tient dans cette conscience de la multitude inéluctable et de l'épreuve de la violence. Les dons surnaturels de Saleem, sa télépathie, son odorat exceptionnellement sensible ainsi que son interprétation de l'histoire en termes d'aliments, de "marinisation" du temps et de l'histoire ("the chutnification of history; the grand hope of the pickling of timen) sont autant de signes-outils pour le déchiffrement du sens. Les emboîtements complexes de la structure narrative permettent de suivre le sens à travers des univers hétérogènes - historiques, sociaux, ethniques, mythologiques, religieux, politiques, littéraires - mais qui sont métonymiquement situés et superposés les uns par rapport aux autres. À un autre niveau de l'organisation des signes qui saturent l'univers romanesque de Rushdie, ce qui se joue constamment dans Midnight's Cbildren, c'est, d'une part, le foisonnement des récits allégoriques (celui des enfants nés le jour de l'indépendance de l'Inde, ceux de la famille, de la mère, du père, du fils, de la pureté, de la patrie, de l'histoire, de la religion, etc.) et, d'autre part, la désallégorisation du monde, le processus de la démythologisation ("Entmythologiesierung"). En tant que roman initiatique, Midnigbt's Children offre donc un champ narratif qui démontre la puissance et la vanité du mythe.

À l'approche du Jour de l'Indépendance, le narrateur réalise que le mythe de la liberté, vieux de 31 ans, n'est plus ce qu'il était. Et il ajoute: "Il faut de nouveaux mythes; mais ce n'est plus mon affaire" (New myths are needed; but that's none of my business"). Saleem se débarrasse de son obsession de la pureté:

5 Salman Rushdie, Midnight's Children, New York, Penguin Books, 1980, p. 551 .

$6 \quad$ Ibid., p. 552. 
106

"Nous devons vivre, je le regrette, avec les ombres de l'imperfection". En clair, nous devons vivre avec les problèmes que notre histoire nous a legués. Dix ans après la parution des Enfants de minuit, Salman Rushdie s'est expliqué sur Les versets sataniques. Son univers romanesque ne se départit pas de son projet cognitif. Ce qu'il se propose de communiquer dans Les versets sataniques êtait déjà inscrit dans la narration même de Midnight's Children:

Les versets sataniques célèbrent l'hybridité, l'impureté, le mélange, la transformation qui se produit à partir des combinaisons inattendues entre les êtres humains, les cultures, les idées, les politiques, les films, les chansons. Ce roman trouve son plaisir dans la bâtardisation et appréhende l'absolutisme de la Pureté. Le mélange, le méli-mélo, un peu de ceci et un peu de cela, voilà comment la nouveauté arrive dans le monde. C'est la possibilité immense qu'offre au monde la grande migration et j’ai essayé de l'accueillir à bras ouverts. Les versets sataniques préconisent le changement-par-fusion, le changement-par-conjonction. C'est un chant d'amour adressé à nos moi(s) bâtards. ${ }^{7}$

Célébrer la bâtardise ne va pas sans quelques difficultés. Saleem traverse de nombreuses incarnations dont celle du bouddha avant d'arriver à la conclusion que le mélange et le changement par fusion peuvent libérer l'Inde de ses conflits.

Ayant parcouru les cycles de l'espoir et de la désillusion, ayant démythifié le pouvoir et la religion, l'idéologie et la nostalgie de la pureté, la narration de Saleem fait marche arrière. Le roman débouche sur une reconnaissance nécessaire de la totalité, réceptacle naturel du multiple. La résistance du pur est vaincue par l'omniprésence de l'impur et de l'hybride. L'immensité de l'Inde impose des modes de vie où l'on doit s'accoutumer à toutes les altérités. Opposé à la force annihilante des croyances et des idéologies en vigueur, opposé à la violence du pouvoir, le geste romanesque de Rushdie est une voie d'espoir.

\section{Carlos Fuentes: Christophe et son ceuf}

Cbristophe et son cuf est un monologue intense, posé de façon grotesque et humoristique par la voix d'un narrateur qui parle depuis le ventre de sa mère pendant les neuf mois de sa gestation, à savoir du 6 janvier au 12 octobre 1992. Le roman de

7 Salman Rushdie, "In good faith", op. cit., p. 394. 
Fuentes est pensé comme un rapport discursif à la totalité mexicaine et, métonymiquement, à la totalité du monde contemporain en voie d'américanisation. Cette totalité est historique, politique, mythique, économique, écologique et littéraire. La voix narrative du fotus est à la fois omnisciente et porteuse d'une vision subjective. Ce partage de compétences produit une sorte de perception symétrique de ce que communique le narrateur (qui de facto ne peut être autre que l'auteur lui-même). La voix du narrateur se superpose à celle de Cristobal, le foetus, et à ce que celui-ci rapporte du dialogue discontinu de ses parents. Le travail discursif sur lequel se fonde le monologue de Cristobal et celui du narrateur omniscient (l'auteur) se constitue en une multitude de séquences et de registres: narratif, incantatoire, lyrique, intertextuel, ludico-langagier. Le monologue de Cristobal s'inscrit en quelque sorte dans la narration du roman que l'on pourrait qualifier de narration-cadre. Celle-ci est, semble-t-il, investie d'un savoir absolu, mais néanmoins consciente de ses limites car, tout en brossant un portrait féroce du pays dans lequel naîtra prochainement Cristobal, le narrateur admet qu'aucune vision ne peut englober tous les phénomènes à la fois :

L'observation simultanée de la totalité des phénomènes est impossible: nous sommes obligés de choisir un temps et un espace à l'intérieur du vaste continuum qu'il nous est donné d'imaginer parce qu'il existe réellement: le morceau de l'ensemble du phénomène qui nous est dévolu constitue notre limite, mais aussi notre liberté: c'est ce sur quoi nous pouvons agir, pour le meilleur ou pour le pire; ce que nous pouvons voir, toucher, ne représente qu'une face de la réalité [...]

nous dépendons de la vision de l'autre pour compléter notre propre vision: nous sommes demi-œil, demi bouche, demi-cerveau, demi-visage; l'autre est moi parce qu'il me complète : [...]. ${ }^{8}$

La vision du réel organisée par Fuentes repose sur un triple rapport sous-jacent à la situation de communication du roman: le "je" de Cristobal et du narrateur omniscient s'oppose à la totalité du narré, qui s'oppose à son tour au lecteur régulièrement interpellé par la narration. En fait, le lecteur devient "électeur". Le lecteur est électeur en tant que citoyen de la République mexicaine. Electeur encore, car il décide du sens du texte. Son interpellation

8 Carlos Fuentes, Christopbe et son ceuf, tr. C. Zins, Paris, Gallimard, 1990, p. 759 . 
108

systématique engendre un jeu cognitif qui relève de ce qu'on pourrait qualifier de "dialogisme orienté". Ce n'est pas tout à fait la même chose que le dialogisme bakhtinien. Le dialogisme orienté s'apparente davantage à la maieutique socratique. Il présuppose une analyse de la réalité, une distribution des rôles dans l'interlocution ainsi qu'une distance ironique. Le dialogisme orienté engage à part entière l'Intelligence Centrale du roman. Un pacte de participation s'établit entre l'auteur-narrateur et le lecteur, comme on le voit dans cette remarque à la fin du roman:

Sapristi, Electeurs! ce sont mes Grands-Parents, ceux du Taco Inépuisable, qui me racontent tout ça, à travers la lignée de mes gènes, à moins que ce ne soit depuis le sonar en forme de gourde noire qui brille à la pointe du mât le plus haut de la Nef de Chine et par ailleurs la situation est la suivante: d'un côté, les Enfants Perdus nous demandent instamment pour la dernière fois, vous venez ou vous ne venez pas? ${ }^{9}$

Entre l'auteur-narrateur et le lecteur-électeur s'instaure une coopération communicative qui garantit la progression de la narration et de la lecture. Le chapitre JE NE VEUX PLUS SERVIR offre un métadiscours particulièrement significatif:

Ceci est le roman que je suis en train d'imaginer à l'intérieur de l'œuf de ma mère. Je n'allais quand même pas faire moins que l'ouf le copain de mes parents. Il s'en faut de beaucoup, mon Christophounet: si la terre est ronde, pourquoi un récit ne le serait-il pas? La ligne droite est la plus longue distance d'un mot à un autre. Mais je sais que je suis une voix clamant dans le désert et que la voix de l'histoire est toujours sur le point de réduire la mienne au silence. ${ }^{10}$

Dans ce métadiscours, le narrateur Cristobal sollicite la collaboration du lecteur-électeur à une démarche narrative dont la fonction prmière est de témoigner de la réalité mexicaine. Voici donc ce que sera le lecteur:

un auxiliaire, un chroniqueur externe respectueux de la consciencieuse enquête sur ma gestation intérieure et de ce qui s'est passé avant elle, car il n'est pas d'événement qui ne soit accompagné de son cortège de souvenirs: en cela nous nous ressemblons toi et moi, Electeur, tous deux nous nous souvenons, moi

$9 \quad$ Ibid., p. 780-781.

10 Ibid., p. 291. 
en synthonie avec ma chaîne génétique et ma natation utérine, toi avec ta présence, ancienne ou récente, au monde extérieur au mien: ce que je ne saurais remémorer, tu le remémoreras pour moi; tu sais ce qui s'est passé, tu ne me laisseras pas mentir, tu te souviens et me racontes que/ ${ }^{11}$

Voici deux autres marques significatives de cette coopération choisies parmi beaucoup d'autres. Au début du roman, dans le chapitre PATRIE, TON TERRITOIRE AMPUTE, le narrateur interpelle les lecteurs-électeurs de la façon suivante:

Prenez l'air Vos Élus et poursuivez le récit que mon père est en train de faire à ma mère le jour de l'Épiphanie tandis qu'ils se nettoient de la merde tombée du ciel et que tous deux, je crois, s'apprêtent à me raconter tout ce qui conduit à cet instant qui est celui qui suit immédiatement mon arrimage $[\ldots]^{12}$

Et dans le chapitre 5 de la septième partie ACCIDENTS DE LA TRIBU, l'interpellation du lecteur prend cette forme :

Voilà pourquoi je te demande, Électeur: Ne nous abandonne pas, maintenant moins que jamais! Pense que ta lecture est notre seule compagnie, notre unique consolation! Nous pouvons tout supporter si tu nous tiens la main! Ne sois pas cruel! Continue à lire. ${ }^{13}$

L'Intelligence Centrale veille à ce que la narration progresse parallèlement à la lecture. Ce couplage est d'ordre axiologique. L'auteur-narrateur construit un univers qui met en évidence des phénomènes observés et choisis dans un espace-temps particulier, celui du Mexique. Cette démarche se double d'une argumentation visant à dénoncer l'ordre établi. La quête de l'auteur-narrateur est celle de "la douce patrie invisible" à laquelle il veut associer les (é)lecteurs. Comme le roman de Fuentes est un espace rempli d'interrogations, il est primordial que ces derniers s'emploient eux aussi à dégager le sens de ce chaos.

Tout au long de son parcours textuel, Christopbe et son ouf est informé d'une structure signifiante du jeu langagier. La narration inscrit dans son corps même un ludisme verbal dont une des fonctions est d'interroger la langue parlée par la communauté mexicaine et donc aussi par Cristobal: 
110

- Segundo: quelle langue va-t-il parler?

- Mais l'espagnol, non?

- Être tous ces nouveaux jargons? L'espanglais, l'anglognol et l'anglaztec inventé par nos copains les Four Foutus et...

- Et le parler de notre amie chilienne Concha Toro, et le francognol de la cabaretière française Ada Ching. Angeles ma chérie, rends-toi compte que nous vivons dans une arène où s'affrontent toutes les langues. ${ }^{14}$

Ce jeu langagier incarne le métissage des mythes, des cultures et des intertextes. En voici deux exemples caractéristiques:

Et mon père se remet à sucer les tétons comme des sucettes, au rythme même de la respiration: Air, Héra, Air, Eros, Aura, Air, Héros, Hair, Hair, Angeles, Shéhérazade, Compteuse Pubique Patentée, Première Romancière, enfonce-toi dans les eaux du temps, trempe-toi le troufignon mon amour, croupe de mon amour angélique $[. .],.{ }^{15}$

Homère, ô mère, ô mar, ô mer, ô merde origine des dieux : thalassa, thalassa. ${ }^{16}$

Ainsi, l'hybride se joue comme une remise en question ironique de la contamination de l'espagnol mexicain par l'anglais américain, mais aussi comme rapprochement d'éléments hétérogènes. Par ailleurs, comme le remarque à juste titre Guy Scarpetta :

ce jeu répond aussi à une civilisation où tout est, depuis la Conquête, hybride, métissé, - et cette façon de "corrompre "l'espagnol revient aussi à assumer (jusqu'à la surenchère) cette situation fondamentale de polyculture, d'impureté. C'est même là tout ce qui fait la portée subversive d'un tel jeu: puisqu'il s'agit en somme d'introduire au coeur de la langue (soit de l'instance symbolique qui fonde et garantit les identifications) quelque chose comme une jouissance de la bâtardise, - ce qui ne manque pas d'apparaître comme la négation la plus éclatante qui soit (et cela excède largement le pur domaine "formel") de tous les fantasmes et de toutes les idéologies de purification. ${ }^{17}$

La narration de Christopbe et son $\propto u f$ renvoie tout au long de son trajet aux expériences collectives du peuple mexicain. En

\footnotetext{
14 Ibid., p. 36.

15 Ibid., p. 23.

16 Ibid., p. 29.

17 Guy Scarpetta, op. cit., p. 324.
} 
cela le roman construit sa dimension symbolique, dialogique et cognitive. Il s'attaque à des problèmes incontournables qui impliquent toute la collectivité mexicaine. Ce renvoi à la "question mexicaine" est réaliste, historique, mais il est aussi iconoclaste, férocement critique. Il constitue un des opérateurs de la modernité du roman. La modernité de Christopbe et son ceuf réside justement dans le fait que sa structure formelle et discursive ainsi que son message sont en prise sur l'actualité immédiate, mais en même temps immergés dans le passé et indexés sur le futur. Le roman de Fuentes se présente alors comme un dispositif textuel complexe, foncièrement rhizomatique, qui cherche à potentialiser voire à assurer l'échange cognitif entre la communauté des lecteurs et le narrateur-messager du roman, porteur d'une vision problématisée du réel. En cela consiste sa modernité.

\section{Edouard Glissant : Tout-monde}

Voici une des définitions de la créolisation qui parsèment l'œuvre théorique de Glissant:

La créolisation exige que les éléments hétérogènes mis en relation "s'intervalorisent", c'est-à-dire qu'il n'y ait pas de dégradation de l'être, soit de l'intérieur, soit de l'extérieur, dans ce contact et dans ce mélange. Et pourquoi la créolisation et pas le métissage? Parce que la créolisation est imprévisible alors que l'on pourrait calculer les effets d'un métissage. ${ }^{18}$

L'œuvre de Glissant est à la mesure de ses considérations critiques et théoriques sur l'écriture romanesque. Il la pense en termes de poétique, de relation, de chaos et de divers. L'hybride participe de cet univers sous forme de créolisation: mélange, conjonction, dynamique d'un ensemble naturel qui reflète la pulsation même de la matière langagière et la rencontre des cultures. L'hybride est en ce sens un opérateur de la totalité, car, pour Glissant, dans son espace réflexif, la créolisation s'ouvre sur le tout du monde de même que celui-ci est inconcevable sans une présence de tous les peuples, du "tout-monde":

je rêve une nouvelle approche, une nouvelle appréciation de la littérature, de la littérature comme découverte du monde,

18 Édouard Glissant, Introduction à une Poétique du Divers, Montréal, Presses de l'Université de Montréal, 1995, p. 16. 
comme découverte du Tout-monde. Je pense que tous les peuples d'aujourd'hui ont une présence importante à assumer dans le non-système de relations du Tout-monde, et qu'un peuple qui n'a pas les moyens de réfléchir à cette fonction est en effet un peuple opprimé, un peuple maintenu en état d'infirmité. Et alors je rêve, pour ma part, puisque je suis un écrivain, je rêve une nouvelle approche de la littérature dans cette démesure qu'est le Tout-monde. ${ }^{19}$

La vocation de Tout-monde est par excellence celle de la relation et de la totalité. Le tout du monde, c'est la traversée du chaos et du divers, éléments autour desquels Glissant organise son discours. Pour Glissant, le chaos-monde c'est le "choc, l'intrication, les répulsions, les attirances, les connivences, les oppositions, les conflits entre les cultures des peuples dans la totalitémonde contemporaine". Le chaos-monde est un "mélange culturel" et non un "simple melting pot par lequel la totalitémonde se trouve aujourd'hui réalisée " 20 . Glissant peut donc être considéré à bon droit comme un auteur-narrateur du "mélange culturel", de la "relation " et du "divers". Ces termes qui lui sont chers permettent de comprendre le sens des configurations formelles et thématiques qui structurent ses cuvres comme une polyphonie de relations humaines immergées dans l'histoire cauchemardesque aussi bien que dans l'utopie d'un monde fraternel.

Ce que Glissant narrativise, met en discours et problématise dans Tout-monde a été pensé en profondeur, théorisé et corrélé par rapport à ce roman. Ainsi, fondamentalement l'idée de la relation. Glissant part des axiomes suivants que nous choisissons dans son livre Poétique de la Relation:

C'est que la pensée de l'errance est aussi bien pensée du relatif, qui est relayé mais aussi relaté. La pensée de l'errance est une poétique, et qui sous-entend qu'à un moment elle se dit. Le dit de l'errance est celui de la Relation. ${ }^{21}$

Dans le système de cette pensée, la relation, l'errance, la créolisation, la totalité et la question de l'identité vont ensemble. La démarche critique et la démarche romanesque de Glissant visent à redéfinir et à problématiser une nouvelle identité qui se dégagerait de la "déstructure des compacités nationales". Selon

19 Ibid., p. 68.

20 Ibid., p. 62.

21 Édouard Glissant, Poétique de la Relation, Paris, Gallimard,1990, p. 31. 
Glissant la recherche de la totalité vue dans le contexte "non universel des histoires de l'Occident, est passée par les stades suivants: pensée du territoire et de soi (ontologique, duelle), pensée du voyage et de l'autre (mécanique, multiple) et pensée de l'errance et de la totalité (relationnelle, dialectique) ${ }^{22}$. Conçu par Glissant comme une forme adéquate à cette quête de la totalité, le roman est l'art de rendre compte du relationnel et du chaotique, du divers et du rhizomatique ou encore l'art d'englober l'hybride.

Tout-monde est un roman cosmique. Il s'agit non seulement du monde entier, mais aussi et surtout de la Caraïbe et de la Martinique ainsi que du Sud dans ce discours à la fois savant et spontanénement populaire, narrativement recherché avec ses doublesfonds, ses récits spéculaires de mythes, d'histoires et de récits déjà advenus illo tempore. C'est le monde d'un "tout" habité par un foisonnement de personnages, de langues, d'idées, de références, d'espaces, de temps historiques et narratifs. Le perspectivisme de Glissant départage et unit tout à la fois les personnages et les espaces, les histoires et les idées, les sentences et les langues, les pays et les territoires, les mythes et les événements, l'histoire et le présent. L'espace textuel se constitue en discontinuité de récits et de discours qui aboutissent à un vertige perspectiviste et totalisant de ce qui est raconté et représenté.

La totalité, tout comme l'errance, la relation, le chaos et le divers peuvent être reconnus comme opérateurs de la modernité. La symbiose qui s'instaure chez Glissant entre la pensée théorisante et la création romanesque mène à une réécriture de la modernité, tout comme elle garantit le renouvellement du roman à la fois dans sa forme et dans ses messages.

\section{Réécriture de la modernité et modernités du roman}

Les distinctions et les lignes de démarcation qui président à la critique et à la théorie littéraires d'aujourd'hui opposent fréquemment roman "moderne" et "postmoderne", si ce n'est roman "moderniste" et "postmoderniste". Par delà les oppositions tranchantes, il serait utile de voir quelles sont les dynamiques et les stratégies du roman au $\mathrm{xx}^{\mathrm{e}}$ siècle. Avançons l'hypothèse que 
114

celles-ci relativisent considérablement les définitions englobantes et exclusives de la modernité et du postmodernisme. Il est essentiel d'identifier les dynamiques et les stratégies propres au roman si l'on veut constituer, sur les plans théorique, historique, discursif et textuel, la toile de fond indispensable à la saisie du sens de ses modernités. Le roman est un genre transculturel, transnational, interdiscursif et intersémiotique. Il traverse différentes cultures sans que celles-ci aient nécessairement des points communs. Dans son développement il transgresse les frontières nationales et celles des langues. Il s'écrit à partir de discursivités qui partagent des éléments communs: rapport entre récit et narrateur, signes du temps et de l'espace ou personnages-"egos expérimentaux" selon la juste formule de Kundera. Le genre romanesque est enfin intersémiotique dans la mesure où il est régi par la présence nécessaire des systèmes modélisants secondaires. Le roman est sémiotiquement déterminé par la coprésence signifiante de structures telles que l'idéologie, l'esthétique, les références ou l'intertextualité. Et tout comme la modernité, le roman opère à l'échelle du monde. Danilo Kis saisit bien ces caractéristiques et ces fonctions du roman:

un genre unique et global, en quelque sorte, "le confluent de tous les genres", il intègre tous les autres types de textes, en empiétant hardiment sur le domaine de la poésie, du drame et surtout de l'essai, et toute tentative de délimitation conduirait (conduit) à de vaines énumérations scolastiques. [...] l'étonnante prolifération du roman européen (et américain) après 1900 place les chercheurs, même les plus scolastiques, devant un problème insoluble: ou bien renoncer à chercher une définition et à procéder à toujours plus de partages, ou bien en arriver à des sous-espèces et des subdivisions cabalistiques. Jamais définitives ni irrévocables de surcroît. En effet, l'on peut dire que chaque nouveau roman important remet en question et modifie fondamentalement la définition du genre luimême. ${ }^{23}$

$\mathrm{Si}$ le roman est devenu un genre-synthèse, il est paradoxal de voir que la dynamique naturelle de ce genre englobant lui permet aussi de durer en tant que forme ouverte, de se transformer et d'établir un équilibre entre l'innovation et la tradition. Autrement dit, le roman est moderne par nécessité organique : sa dynamique d'ouverture protéiforme et son potentiel d'inclusion dialec-

23 Danilo Kis, Homo poeticus, tr. P. Delpech, Paris, Fayard, 1993, p. 106-107. 
tique de "corps étrangers" dans son propre corps textuel garantissent l'évolution du genre. La forme romanesque évolue vers un état de choses à la fois transitoire et stable où l'impératif du moderne n'est rien d'autre que la réalisation du postulat de la pertinence cognitive du roman. Surgit alors le problème de la réécriture de la modernité. Les opérateurs de la modernité sont variables, mais leur telos est en principe toujours le même. C'est l'inéluctabilité de l'épreuve cognitive, d'un nouveau positionnement des signes. Réécrire la modernité est en quelque sorte présupposé par la spécificité discursive de la forme romanesque, par son polymorphisme et sa disponibilité à accueillir presque tous les discours, quel que soit leur lieu énonciatif d'origine. Le roman "met à l'essai des discours " ${ }^{24}$ qu'il intègre intentionnellement pour pratiquer une sorte d'expérimentation scientifique. En ce sens, il lui est relativement facile de réécrire la modernité puisque sa forme est par définition inachevée, expérimentale, en perpétuel processus d'ajustement et de redéfinition par rapport à la complexité du réel. En commentant les expériences discursives que pratiquait Robert Musil, Walter Moser précise :

Il est vrai que Musil maintient une charpente narrative qui règle l'interaction des personnages d'après le schéma "lorsque ", "avant que", "après que", pourtant l'action représentée dans le roman devient de plus en plus celle d'un faire discursif. Elle se manifeste dans des dialogues, dans des réflexions attribuées ou non à un personnage, dans des citations et allusions, dans des explorations quasi philologiques, dans des critiques faites des différents "langage", complémentaires et concurrentiels, qui se partagent l'explication du monde et de l'homme ainsi que la structuration et le fonctionnement de la société. C'est ainsi que le faire romanesque n'est plus tant une opération mimétique visant à représenter le monde, la société, la vie, qu une mise à l'essai des discours les plus divers. ${ }^{25}$

Le modèle musilien acquiert une place considérable dans le roman $\mathrm{du} \mathrm{xx}^{\mathrm{e}}$ siècle, car il potentialise l'ouverture, la mä̈eutique, le fonctionnement d'un dispositif ironique et la dialectique thématico-formelle du genre dont celui-ci a de plus en plus besoin pour répondre aux défis immédiats du réel, du social et

24 Walter Moser, "Ia mise à l'essai des discours dans L'Homme sans qualités de Robert Musil ", Novels and Intertexts II. Canadian Review of Comparative Literature (W. Krysinski (éd.)), mars 1985, p. 12-45. 
du politique. Ainsi le roman devient-t-il de plus en plus expérimental et ouvert, discours du possible sans frontières déterminées. Nous l'avons vu dans le cas d'une série de romanciers qui, chacun à sa façon, déstabilisent et restabilisent le roman en perfectionnant son écriture. Dans l'esprit de Bakhtine, le roman est apte à relever le défi de l'épreuve cognitive que lui pose le réel puisqu'il est la forme la plus proche de l'immédiat et du présent. Cette forme active une combinatoire des problématiques auxquelles l'œuvre se réfère et qu'elle soumet à un traitement narratif et discursif afin de répondre aux interrogations posées par le réel et le social.

Définir les modernités du roman présuppose une mise en perspective de la modernité même et de ses réécritures ${ }^{26}$. Lyotard relativise considérablement le sens de l'opposition entre modernité et postmodernisme:

Une œuvre ne peut devenir moderne que si elle est d'abord postmoderne. Le postmodernisme ainsi entendu n'est pas le modernisme à sa fin, mais à l'état naissant, et cet état est constant. ${ }^{27}$

La postmodernité n'est pas un âge nouveau, c'est la réécriture de quelques traits revendiqués par la modernité, et d'abord de sa prétention à fonder sa légitimité sur le projet d'émancipation de l'humanité tout entière par la science et la technique. Mais cette réécriture, je l'ai dit, est à l'œuvre, depuis longtemps déjà, dans la modernité elle-même. ${ }^{28}$

La modernité s'écrit, s'inscrit sur elle-même, en une perpétuelle ré-écriture. ${ }^{29}$

26 Il est évident que ces mises en perspective peuvent et même doivent se faire à partir de données historiques. Ainsi a-t-on circonscrit historiquement avec beaucoup de justesse le "high modernism. ou le code du modernisme. Voir en particulier les travaux de J.-M. Rabaté ("Introduction au modernisme historique ", Moderne, Modernité, Modernisme. Les Cabiers du Musée National d'Art Moderne, 19-20, Juin 1987, p.94-108) et de D. Fokkema ("A Semiotic Definition of Aesthetic Experience and the Period Code of Modernism", Types of the Novel, Semiotics of Social Discourse. Poetics Today, 3: 1, Hiver 1982, p. 61-79). Voir aussi G. Cianci (éd.), Modernismo / Modernismi, dall'avanguardia storica agli anni Trenta e oltre, Milano, Ed. Principato, 1991.

27 Jean-François Lyotard, Le postmoderne expliqué aux enfants, Paris, Galilée, 1986, p. 30.

28 Jean-François Lyotard, "Réécrire la modernitée, L'inbumain. Causeries sur le temps, Paris, Galilée, 1988, p. 43.

29 Ibid., p. 37. 
De quelle façon cette position théorique permet-elle de problématiser la question des modernités du roman? Lyotard affirme que la modernité est un processus ininterrompu, qu'elle se réécrit sans cesse. En affirmant que le moderne se renouvelle par le postmoderne, il donne un nouveau rang discursif au postmodernisme. Ainsi, dans son élan créateur, le roman, se réalise par la réécriture de certains éléments thématiques et formels afin d'accomplir le postulat de la pertinence cognitive. La réécriture de la modernité est une catégorie calquée sur le concept freudien de "Durcharbeitung" - perlaboration, travail de remémoration constructive, anamnèse où entre du refoulement et qui est hantée par la "langue", la tradition, le matériau avec, contre et dans lequel on écrit " ${ }^{30}$. La réécriture de la modernité du roman est donc une réécriture dialectiquement orientée de la langue et de la tradition ainsi que des matériaux mêmes. Nous avons essayé de problématiser la modernité du roman en soulignant qu'elle est un fait pluriel qui s'accomplit à l'échelle du genre tout entier et dans chaque cuvre singulière. La modernité du roman est un fait pluriel dans la mesure où elle se réalise par la mise en jeu dialectique de certains éléments prévalants, et non comme une "modernisation" massive de la forme romanesque proprement dite que l'on retrouverait dans chaque roman identifié comme "moderne". Ainsi pouvons-nous mettre en relief les faits suivants: 1) la modernité du roman se déploie sur une longue durée historique en tant que processus continu et dialectiquement marqué; 2) les opérateurs de la modernité (subjectivité, fragmentation, discontinuité, ironie, auto-réflexivité) agissent comme filtres discursifs imprimant à la forme romanesque une ou plusieurs spécificités du "moderne"; 3) le roman est un genre hautement interdiscursif et intertextuel de sorte que ses modèles matriciels communiquent; 4) le roman est parmi tous les genres littéraires le plus proche du présent et possède de ce fait un très grand potentiel d'adaptation thématique et formel au réel et au social. Par là même, le roman réécrit ses modernités chaque fois qu'il s'engage dans le processus cognitif.

La position nuancée de Lyotard nous permet d'englober le postmodernisme ou plutôt le moment postmoderne du roman dans le processus de réécriture de la modernité. Il s'ensuit que les oppositions tranchantes qui départagent systématiquement le 
118

postmodernisme de la modernité, selon des critères aussi incertains que divergents, s'avèrent caduques ${ }^{31}$. La réécriture de la modernité présuppose un rapport actif, fonctionnel et créateur du postmoderne au moderne. La spécificité de la forme romanesque que nous avons décrite en termes de malléabilité, de disponibilité et d'ouverture octroie au discours romanesque la qualité d'instru-

31 Voir à ce propos les considérations de Linda Hutcheon: "Postmodern theory, criticism, and art today are all engaged in contesting the modernist (humanist) premises of art's apolitical autonomy and of theory and criticism as value-free activities. The postmodern paradoxes both reveal and question prevailing norms, and they can do so because they incarnate both processes." Linda Hutcheon, A Poetics of Postmodernism, New York et Londres, Routledge, 1988 , p. 230.

Cette exclusivité présuppose une division et une classification qui départagent le moderne du postmoderne. Or, l'attribution de cette exclusivité au postmodernisme est discutable. Maints artistes et critiques reconnus comme "modernes" ont contesté l'idée de l'apolitisme de l'art. Brian McHale définit la différence entre la fiction moderniste et postmoderniste de la façon suivante: "I will formulate it as a general thesis about modemist fiction; the dominant of modernist fiction is epistemological. That is, modernist fiction deploys strategies which engage and foreground questions such as those mentioned by Dick Higgins in my epigraph:" How can I interpret this world of which I am a part? And what am I in it?" [...] This brings me to a second general thesis, this time about postmodernist fiction: the dominant of postmodern fiction is ontological. That is, postmodernist fiction deploys strategies which engage and foreground questions like the ones Dick Higgins calls "post-cognitive": "Which world is this? What is to be done in it? Which of my selves is to do it?" ", B. McHale, Postmodernist Fiction, New York et Londres, Routledge, 1993 (1987), p. 9-10. Là encore, bien que posée en termes de "dominantes", l'opposition est marquée entre ce qui est épistémologique et ce qui est ontologique dans la fiction moderniste et postmoderniste. Or il est évident que la fiction moderniste peut, elle aussi, avoir des interrogations de type ontologique de même que la fiction postmoderniste ne tourne pas tout à fait le dos à des questionnements de nature épistémologique.

$\mathrm{K}$. Beekman remet en cause la validité de la notion même de "postmoderne" utilisée à des fins de classification: "The designation "postmoderne prose" in its turn lost its classifying force because, failing to provide the necessary and sufficient conditions for classifying texts as "postmodern", such a large number of texts from such different periods as the 60 s and the 80 s were labeled "postmodern", that subdivisions became inevitable. Strangely enough, in making these, scholars fell back on the old critized categories. [...] So the theoretical status of the concept of "postmodern prose" is questionable. Until now it has remained unclear whether it is a generic term or not, what the sufficient and necessary conditions are to classify a phenomenon as postmodern and how the assumed characteristics can be analyzed in an unambiguous way", K. Beekman, "Changing Labels: From "Experimental" to "Postmodern" Prose * in Institution E Innovation. Avant Garde Critical Studies, 8, Amsterdam-Atlanta, Rodopi, 1994, p. 138. 
ment cognitif: les modernités du roman sont liées à la réalisation de la pertinence cognitive la plus appropriée dans les contextes formels et thématiques précis où surgit un roman donné.

En admettant que les modernités du roman se jouent sur une scène planétaire, les séries pour lesquelles nous avons opté illustrent le sens de la progression du roman et de son implication dans le processus cognitif. Dans ces différentes séries, la réécriture de la modernité précise la nature des problèmes, des traits et des stratégies dites "modernes". C'est ainsi que les modernités du roman que nous voulons saisir se manifestent par une mosaïque de signes qui dénotent ou connotent, d'une part, la diversité et la fluctuation des thèmes et des formes et, d'autre part, la pertinence cognitive des structures discursives engagées dans le processus de communication. Les modernités du roman que nous avons mises en évidence renvoient à différentes époques du roman, mais en même temps, elles confirment l'extraordinaire continuité du genre. Voici quelques-unes des structures saillantes de la modernité telle que les romans inscrits dans notre champ critique l'ont thématisée et réécrite :

1. Joyce, Musil, Witkiewicz, Broch, Cortazar: Les œuvres de ces romanciers se caractérisent par la révolution ("permanente") du langage romanesque, la redéfinition de nouveaux paramètres discursifs propres au roman et par la construction d'un espace romanesque autonome. La totalité y apparaît comme un opérateur axiologique du discours romanesque ${ }^{32}$. Ces œuvres déploient un nouvel espace-temps; elles sont marquées par le polylinguisme, l'hybridation des langages, le poétique, le sens du

32 Les observations suivantes de Pierre Fougeyrollas s'appliquent bien à la compréhension des devenirs et des finalités du roman: "La totalité, sous quelques espèces qu'on la conçoive, est une affirmation de l'absolu comme négation du relatif et du partiel. Par là, la totalité se révèle comme une manifestation de la négativité . P. Fougeyrollas, Contradiction et totalité, Sungissement et déploiements de la dialectique, Paris, Minuit, 1964, p. 16.

Le roman est une forme symbolique et dialectique dans la mesure où il est régi par des rapports multiples au relatif, au partiel et à l'absolu. Dialectiquement, les visées de la totalité dans le champ du relatif et du partiel, constituent la forme romanesque en une négativité (*Aufhebung») et permettent de déclencher le processus d'opposition des discours dont l'issue implique une "manifestation de la négativé ". Nicolas Rosa saisit ce problème avec justesse en définissant le lieu de Marelle dans la tradition du roman moderne. Cf. N. Rosa, Los fulgores del simulacro, Universidad Nacional del Litoral, 1987, p. 179. 
métatexte et du ludique et la mise en place d'un dispositif ironique. Elles mobilisent la participation active du lecteur au titre de créateur du roman, comme dans Marelle ${ }^{33}$.

2. Lins, Manganelli, Roa-Bastos, Wolf, Andrzejewski, Kundera: Les romans qui se caractérisent ici par l'art de la composition marquent le retour du métadiscursif et sa redéfinition. Dans la lignée du "métatexte viennois", ces cuvres remettent en cause le métatexte comme pur jeu textuel. Le métatexte et la métafiction sont davantage branchés sur la vie, sur l'histoire et sur le sens ontologique du littéraire.

3. Fuentes, Rusbdie, Glissant, Boudjedra, Chamoiseau: Avec ces romanciers, la périphérie s'inscrit dans le corpus global du roman, et avec elle l'hybridisation, la créolisation, la déstabilisation du grand canon occidental au profit de la mise en valeur du "tout-monde".

4. Saramago, Goytisolo, Balestrini, Sollers, Galeano, Tabucchi, Semprun: On retrouve chez ces romanciers le rapport à l'histoire, à l'hybride, au politique et à la totalité. Mais c'est au travers de nouvelles écritures romanesques: structures enchâssantes chez Saramago; polylogue et "écriture percurrente" chez Sollers; scansion et prosodie chez Balestrini; "algarabisation" de la prose chez Semprun; interlocution anaphorique chez Tabucchi.

5. Guimaraes Rosa, Lispector, Arenas, Bernbard, de Azua, Llansol, Meddeb, Ben Jelloun, Brodkey, Easton Ellis: Ces œuvres

33 Milagros Ezquerro problématise de façon pertinente la lecture de Marelle. Tout en rappelant que les "catégories kantiennes" (l'espace, le temps, la causalité, la non-contradiction, le principe d'identité), selon l'expression d'un des personnages, sont remises en cause dans ce roman, elle évoque la présence de la "figure symbolique" qui apparaît sous diverses formes tout au long du roman: la marelle, le labyrintbe, l'Igdrassil, le mandala. Marelle construit une réalité romanesque dont les coordonnées structurales sont ambiguës, imprécises, douteuses, branlantes: le temps de la lecture est luimême happé par ce mécanisme déstructurant. À la lecture traditionnelle, cursive, linéaire, sans problèmes, le lecteur est enjoint de substituer une lecture labyrinthique, spasmodique, problématique, ou mieux, une lecture libre, sans principe directeur, entièrement autonome et créatrice de l'espace textuel. Que cette subversion du temps dans Marelle soit la projection d'une vision du monde dont les principes structurants sont radicalement contestés, ce n'est guère douteux; mais l'important c'est précisément que cette vision du monde s'exprime par la destruction de la réalité romanesque dans ses éléments constituants fondamentaux". Milagros Ezquerro, "Théorie et Fiction, le nouveau roman hispano-américain ", Études critiques, 1983, p. 31-32. 
réaccentuent le subjectif. Pulsions et monologues y font retour. Ces textualités pulsionnelles sont aussi marquées par la discontinuité 34 .

Voilà comment les réécritures du roman présupposent l'avènement d'un ensemble de discursivités comprises comme "actions communicatives" et qui s'inscrivent dans le continuum historique du genre. Elles caractérisent l'évolution du roman depuis le début du siècle. Nous estimons qu'il y a lieu de les considérer comme des "actions communicatives" au sens que Jürgen Habermas donne à cette notion lorsque dans sa théorie de l'action communicative, il souligne que les actions sociales et politiques doivent être pensées et accomplies de façon intersubjective ${ }^{35}$. Dans son développement historique et réalisant le postulat du moderne tel que Baudelaire ${ }^{36}$ et Rimbaud l'ont conçu ("Il faut être absolument modernen), le roman s'inscrit par ses stratégies discursives dans une quête cognitive de la "communauté idéale de la parole". Il parle et agit contre le "silence qui est la tragédie politique ultime de la langue " 37 .

L'impératif du moderne lui impose la recherche d'un discours et d'une architecture formelle, conditions nécessaires de sa pertinence cognitive ${ }^{38}$ engagée dans le processus dialectique de la tension entre la tradition et l'innovation.

Les linéaments de l'évolution du roman au $\mathrm{xx}^{\mathrm{e}}$ siècle confirment la pertinence de la théorie bakhtinienne du roman "dialogique" et "polyphonique". Mais ils démontrent par ailleurs que le

34 Pour une une mise au point importante sur le subjectif dans le roman moderne, voir J.-M. Bernstein, The Philosophy of the Novel. Lukacs, Marxism And the Dialectics of Form, Minneapolis, Univerity of Minnesota Press, 1984.

35 J. Forester, "Introduction: The Applied Turn in Contemporary Critical Theory ", Critical Theory and Public Life (J. Forester (ed.)), Cambridge, Massachusetts, The MIT Press, 1987 (1985), p. XI.

36 Voir le commentaire suivant de H.-R. Jauss: "pour Baudelaire l'expérience esthétique et l'expérience historique de la modernité se confondent. [...] $\AA$ l'exemple du romantisme selon Stendhal, la modernité selon Baudelaire, entrainée par le mouvement accéléré de la conscience historique, ne cesse de se redéfinir en opposition à elle-même. ", Literaturgeschichte als Provokation, Frankfurt am Main, Suhrkamp, 1970, p. 55-56.

37 J. O'Neill, "Decolonization and the Ideal Speech Community: Some Issues in the Theory and Practice of Communicative Competence ", in J. Forester, $o p$. cit., p. 57.

38 Voir à cet égard Saul Yurkievich, Julio Cortazar, al calor de tu sombra, Buenos Aires, Editorial Legasa, 1987, p. 23-25. 
roman s'est exprimé et s'exprime encore par des voix monologiques. Il n'est pas question de nier la fonction cognitive du dialogisme et du dialogue en tant que structures épistémologiques opératoires dans le discours romanesque. Il est évident que Bakhtine a eu l'intuition de cette dynamique principale du roman moderne que $M$. Holquist identifie bien tout en insistant sur le fait que Bakhtine diffère fondamentalement de Hegel et de Lukacs:

Au lieu d'une vague unitaire de la conscience qui progresse et s'élève constamment comme nous la trouvons chez Hegel et chez Lukacs, le dialogisme conçoit l'histoire comme une lutte permanente entre le monologue et le dialogue avec la possibilité toujours présente de renversements. Le roman est le texte caractéristique d'un stade particulier dans l'histoire de la conscience non pas parce qu'il marque la découverte du moi par lui-même ("the self's discovery of itself "), mais parce qu'il manifeste la découverte de l'autre par le moi ("the self's discovery of the other "). ${ }^{39}$

Cela dit, en observant sa progression depuis Cervantes, Sterne, Diderot ou Dostoïevski jusqu'à Biely, Beckett, Lispector ou Arenas, on voit que le roman ne cesse de confirmer la présence énonciative, puissante et parfois dominante, du monologisme. Ce dernier s'inscrit dans ce que Michael André Bernstein appelle à juste titre "la poétique du ressentiment " ${ }^{40}$ qui, de facto, n'entre pas en dialogue, ni ne se prévaut des privilèges du dialogisme, alors que dans l'esprit de Bakhtine celui-ci règne imperturbablement sur toutes les narrations et sur tous les discours. Il s'agirait en l'occurrence de déplacer l'accent théorique pour répondre objectivement aux impératifs du discours homophonique tel qu'il advient effectivement dans le roman postdostoïevskien. On ne peut qu'être d'accord avec Bakhtine, à condition de relativiser son impact sur la théorie actuelle ou actualisante du roman, compte tenu de données objectives.

39 M. Holquist, Dialogism, Bakbtin and bis World, Londres, New York, Routledge, 1990, p. 75. (Nous traduisons). Voir aussi A. Ponzio, Tra semiotica e letteratura, Introduzione a Michail Bacbtin, Milano, Bompiani, 1992; et S. Petrilli, Materia segnica e interpretazione, Lecce, Ed. Milella, 1995, tout particulièrement les chapitres suivants : "Le categorie bachtiniane della letteratura per una nuova filosofia del linguaggio" et "Produzione linguistica, ideologia e alterità : la filosofia del linguagio di Augusto Ponzio *

40 Michael André Bernstein, "The Poetics of Ressentiment ", Retbinking Bakbtin, Extensions and Cballenges, (G.S. Morson and C. Emerson (éds.)), Evanston, Illinois, Northwestern University Press, 1989, p. 197-223. 